\title{
Dose Response Control
}

National Cancer Institute

\section{Source}

National Cancer Institute. Dose Response Control. NCI Thesaurus. Code C120841.

A type of control using different doses or regimens of the same treatment (fixed dose) among the study groups. [From ICH E10]. 